\title{
Comportamentos relacionados à saúde entre participantes e não participantes da ginástica laboral
}

\author{
Health-related behaviors of participants and non-participants \\ in a workplace physical activity program
}

\author{
Antonio José Grande \\ Mathias Roberto Loch \\ Evanil Antonio Guarido \\ João Bruno Yoshinoga Costa ${ }^{2}$ \\ Gabriela Claudino Grande 2 \\ Felipe Fossati Reichert ${ }^{3}$
}

1 Universidade Metodista de Piracicaba. Pós-Graduação em Educação Física. Piracicaba, SP. Brasil.

2 Universidade Estadual de Londrina. Londrina, PR. Brasil

3 Universidade Federal de Pelotas. Pelotas, RS. Brasil

Recebido em 28/09/10 Revisado em 28/10/10 Aprovado em 09/11/10
Resumo - O objetivo do trabalho foi verificar a prevalência de comportamentos relacionados à saúde em trabalhadores participantes e não participantes da Ginástica Laboral (GL) da Universidade Estadual de Londrina. Foram aleatorizados 20 setores do campus universitário participantes do programa de GL. Foram entregues 373 questionários dos quais 334 (89,5\%) retornaram preenchidos. Para a análise dos dados, utilizaram-se a estatística descritiva e o teste do qui-quadrado. Os participantes do programa de GL apresentaram menor prevalência de inatividade física no tempo livre $(49,3 \%)$ e consumo abusivo de álcool $(17,2 \%)$ do que os não participantes $(63,4 \%$ e $25,8 \%$, respectivamente. Entre os homens, os participantes apresentaram menor inatividade física no lazer, frequência de tabagismo e percepção negativa de estresse. Entretanto, o consumo de frutas insuficiente foi menor entre os que não participavam (52,6\% dos não participantes e 72,1\% dos participantes), bem como o consumo insuficiente de verduras (29,9\% dos não participantes, contra 49,2\% dos participantes). As mulheres praticantes do programa de GL referiram menor insatisfação com os colegas de trabalho (2,2\% das que participam, contra 9,3\% das que não participam). Os participantes do programa de GL apresentaram menor prevalência de inatividade física no lazer e de consumo abusivo de álcool, entretanto, nas demais variáveis não se observaram diferenças significativas. Intervenções mais abrangentes devem ser implementadas com o objetivo de diminuir a prevalência de comportamentos de risco entre os trabalhadores.

Palavras-chave: Atividade motora; Estilo de vida; Saúde do trabalhador.

Abstract - The aim of this study was to determine the prevalence of health-related behaviors among workers participating or not in a workplace physical activity program (WPA) at Universidade Estadual de Londrina. Twenty sectors of the university campus participating in the WPA program were randomized. A total of 373 questionnaires were handed out and 334 (89.5\%) completed questionnaires were returned. Descriptive statistics and the chi-square test were used for data analysis. Participants in the program presented a lower prevalence of physical inactivity during leisure time (49.3\%) and alcohol abuse (17.2\%) than non-participants (63.4\% and $25.8 \%$, respectively). The frequency of physical inactivity during leisure time, smoking and negative perception of stress was lower among male participants. However, the frequency of insufficient consumption of fruits (52.6\% of non-participants versus $72.1 \%$ of participants) and vegetables (29.9\% of non-participants versus $49.2 \%$ of participants) was lower among non-participants. Female participants reported less dissatisfaction with work colleagues (2.2\% of participants versus 9.3\% of non-participants.) The prevalence of physical inactivity and alcohol abuse was lower among WPA participants, but no significant differences were observed for the other variables. More comprehensive interventions should be implemented in order to reduce the prevalence of risk behaviors among workers.

Key words: Motor activity; Lifestyle; Occupational health. 


\section{INTRODUÇÃO}

O número de pesquisas com foco em intervenções profissionais no trabalho vem crescendo nos últimos anos ${ }^{1-8}$. Muitos destes trabalhos investigaram o impacto de programas de promoção da saúde no contexto do trabalho.

No cenário internacional, pesquisas recentes com intervenção no ambiente de trabalho vêm destacando o potencial para realização de alterações nos comportamentos relacionados à saúde, o que geraria um impacto positivo na saúde dos trabalhadores e na diminuição dos custos das empresas ${ }^{8-10}$.

No Brasil, uma das formas mais utilizadas de intervenção no trabalho, com intuito de prevenção de doenças e promoção da saúde, é a ginástica laboral $(\mathrm{GL})^{3}$. A GL tem características de preparação muscular para o trabalho, pausa, correção postural ou relaxamento muscular ${ }^{11}$.

Entre os estudos com esta temática, destaca-se que os mesmos investigaram fundamentalmente a função da GL no aumento da produção, na redução do absenteísmo, na prevenção de doenças ocupacionais e na melhoria de qualidade de vida dos trabalhadores ${ }^{1-7}$.

A efetividade das intervenções no local de trabalho é parcialmente dependente dos métodos empregados, dos desfechos investigados e das características de cada contexto. Essas particularidades contribuem para a falta de consistência da literatura em relação ao sucesso das intervenções no ambiente de trabalho. Vale destacar, ainda, que, para alguns, o tempo destinado à prática de GL é insuficiente para alteração de alguns desfechos de saúde - incluindo aí alguns comportamentos relacionados à saúde ${ }^{1,20}$. Entretanto, outros autores acreditam que a GL pode potencialmente ser benéfica ao incentivar os trabalhadores a terem comportamentos positivos também fora do ambiente de trabalho ${ }^{17,20}$.

Evidentemente, não se pode esquecer que a alteração ou não de certos comportamentos depende de muitos outros aspectos, inclusive de ordem social, econômica e ambiental ${ }^{13,21}$.

Neste sentido, o objetivo principal deste trabalho foi comparar os comportamentos relacionados à saúde entre trabalhadores participantes e não participantes da ginástica laboral na Universidade Estadual de Londrina. Como objetivo secundário, investigou-se a prevalência dos comportamentos segundo gênero e nível educacional.

\section{PROCEDIMENTOS METODOLÓGICOS}

A Universidade Estadual de Londrina (UEL) tem um programa de ginástica laboral estabelecido desde 2004. O programa desenvolve quinze minutos de alongamentos diários, desenvolvido por alunos (estagiários) dos cursos do Centro de Educação Física e Esporte da UEL.

Em um primeiro momento, foram levantadas informações junto à coordenação do Programa de GL da UEL, que informou serem 65 os setores e repartições participantes da GL do Campus Universitário. Uma vez identificados os setores que possuem GL, utilizou-se o programa Randomizer (www.randomizer.org), para aleatorização dos 20 setores que seriam sorteados. A escolha de 20 setores para serem investigados foi definida com base em aspectos logísticos, observando-se o prazo que havia para a coleta de dados e o número de pesquisadores participantes. No entanto, a aleatorização da amostra permitiu que todos os setores tivessem a mesma chance de serem sorteados, garantindo, assim, a representatividade da amostra. Participaram da pesquisa, exclusivamente, trabalhadores técnico-administrativos, excluindo-se, assim, estagiários e docentes.

Todos os trabalhadores dos setores sorteados foram convidados a participar do estudo. Para tanto, foram esclarecidos sobre os procedimentos do estudo e assinaram o Termo de Consentimento Livre e Esclarecido.

Foi entregue um questionário, sem necessidade de identificação, com informações demográficas, sobre a participação ou não no Programa de GL e sobre alguns indicadores e comportamentos relacionados à saúde (prática de atividade física, características da alimentação, fumo, consumo abusivo de álcool, percepção sobre a qualidade dos relacionamentos e percepção saúde e de estresse). $\mathrm{O}$ questionário utilizado foi elaborado com perguntas de outros questionários já utilizados em estudos como o do VIGITEL (Vigilância de Doenças Crônicas por Inquérito Telefônico) ${ }^{14} \mathrm{e}$ o do SESI (Serviço Social da Indústria ${ }^{17}$. Os trabalhadores tiveram o período de uma semana para responderem. Durante essa semana, o pesquisador voltou a cada setor para motivar os trabalhadores a preencherem o questionário. Para os trabalhadores com ensino médio incompleto, foram realizadas entrevistas individualizadas, em horários previamente agendados com os próprios trabalhadores e responsáveis pelos setores.

$\mathrm{Na}$ semana seguinte, o pesquisador retornou aos setores para coletar os questionários respondidos, os quais eram colocados em envelopes, misturando-os com outros já respondidos, garantindo o sigilo das informações.

A seguir, seguem as variáveis estudadas e seus respectivos critérios de análise ${ }^{14,17,22}$. 
- Consumo inadequado de frutas e verduras: Consumo em menos de cinco dias em uma semana típica.

- Inatividade física no lazer: Aqueles que não praticavam pelo menos 20 minutos de atividades físicas em três dias de uma semana típica, no tempo livre.

- Tabagismo: Fuma atualmente, independentemente da quantidade.

- Consumo abusivo de álcool: Ingestão de cinco ou mais doses em pelo menos uma ocasião nos últimos 30 dias.

- Percepção negativa de saúde: Percebe sua saúde como regular ou ruim

- Insatisfação no relacionamento com colegas de trabalho: Refere-se insatisfeito ou muito insatisfeito com colegas de trabalho;

- Percepção negativa de estresse: Se percebe como quase sempre ou sempre estressado.

Todos os procedimentos foram aprovados pelo Comitê de Ética em Pesquisa com Seres Humanos da UEL (Parecer 155/09).

Os dados foram tabulados e analisados no SPSS versão 17.0. Foram utilizados elementos da estatística descritiva e o teste do qui-quadrado, usado para verificar eventuais diferenças entre os grupos, adotando-se $\mathrm{p} \leq 0,05$.

\section{RESULTADOS}

Foram entregues 373 questionários aos trabalhadores dos setores sorteados, dos quais 334 (89,5\%) retornaram preenchidos. A média de idade dos trabalhadores investigados foi de 44,3 anos (DP = 9,8 anos). A tabela 1 apresenta detalhes a respeito da composição geral da amostra.
Tabela 1. Características gerais da amostra.

\begin{tabular}{lcc}
\hline Variável & $\begin{array}{c}\text { Freqüência } \\
\mathrm{n}\end{array}$ & $\begin{array}{c}\text { Percentual } \\
\%\end{array}$ \\
\hline Gênero & 158 & 47,3 \\
\hline Masculino & 176 & 52,7 \\
\hline Feminino & & \\
\hline Estado Civil & 83 & 24,9 \\
\hline $\begin{array}{l}\text { Solteiro } \\
\text { Casado }\end{array}$ & 190 & 57,1 \\
\hline $\begin{array}{l}\text { Outro } \\
\text { Escolaridade }\end{array}$ & 60 & 18 \\
\hline $\begin{array}{l}\text { Fundamental completo/Médio } \\
\text { incompleto }\end{array}$ & 41 & 12,3 \\
\hline $\begin{array}{l}\text { Médio completo/superior } \\
\text { incompleto }\end{array}$ & 105 & 31,4 \\
\hline $\begin{array}{l}\text { Superior Completo } \\
\text { Pós-graduado }\end{array}$ & 74 & 22,2 \\
\hline
\end{tabular}

Observa-se, na tabela 1, que a amostra foi composta de maneira equilibrada pelos dois gêneros, com ligeiro predomínio de mulheres. A maior parte referiu ser casada e apresentou bom nível de escolaridade (56,3\% tinham pelo menos nível superior completo). Quanto à participação na GL, mais da metade $(54,8 \%)$ referiu não participar do programa.

A tabela 2 apresenta a prevalência dos comportamentos relacionados à saúde, estratificando por gênero e nível educacional.

Observa-se, na tabela 2, menor participação na ginástica laboral entre os homens. Os homens também apresentaram maior prevalência de consumo insuficiente de frutas e de verduras, além de maior consumo abusivo de álcool. Por outro lado, as mulheres referiram maior percepção negativa de estresse. Nas variáveis inatividade física no lazer, tabagismo e insatisfação com os colegas de trabalho, não se observou diferença significativa entre os gêneros.

Quando estratificada a amostra, segundo nível educacional, observou-se que a prevalência de

Tabela 2. Participação na G.L estratificados pelo gênero e pelo nível educacional.

\begin{tabular}{|c|c|c|c|c|c|c|}
\hline \multirow[b]{2}{*}{ Variável } & \multicolumn{2}{|c|}{ Gênero } & \multirow[b]{2}{*}{$\mathrm{p}$} & \multicolumn{2}{|c|}{ Nível educacional } & \multirow[b]{2}{*}{$\mathrm{p}$} \\
\hline & $\begin{array}{c}\text { Masc. } \\
\%\end{array}$ & $\begin{array}{c}\text { Fem. } \\
\%\end{array}$ & & $\begin{array}{r}\geq \mathrm{SC} \\
\%\end{array}$ & $\begin{array}{c}<\mathrm{SC} \\
\%\end{array}$ & \\
\hline Não participa da GL & 61,4 & 48,9 & 0,014 & 58,0 & 50,7 & 0,112 \\
\hline Consumo insuficiente de frutas & 60,1 & 41,5 & 0,001 & 43,6 & 58,9 & 0,004 \\
\hline Consumo insuficiente de verduras & 37,3 & 23,3 & 0,001 & 23,9 & 38,9 & 0,005 \\
\hline Inatividade física no lazer & 56,3 & 56,8 & 0,508 & 51,6 & 63,0 & 0,024 \\
\hline Fumante & 12,7 & 14,8 & 0,345 & 11,7 & 16,4 & 0,139 \\
\hline Consumo abusivo de álcool & 33,1 & 11,9 & 0,001 & 26,6 & 15,9 & 0,013 \\
\hline Percepção negativa de estresse & 13,5 & 26,1 & 0,03 & 23,4 & 16,0 & 0,06 \\
\hline Insatisfeitos com colegas de trabalho & 2,6 & 5,7 & 0,127 & 5,3 & 2,8 & 0,194 \\
\hline
\end{tabular}

$\geq \mathrm{SC}=$ superior completo ou pós-graduado

$<\mathrm{SC}=$ menor que superior completo 
consumo insuficiente de frutas e de verduras, além da inatividade física no lazer, foi superior entre aqueles com menor nível educacional. No entanto, o consumo de bebidas alcoólicas, foi superior entre aqueles com maior nível educacional. Para as demais variáveis não foi constatada diferença significativa entre os diferentes níveis educacionais investigados.

A Tabela 3 apresenta a prevalência de indicadores de saúde e do estilo de vida, estratificando segundo participação nas sessões de ginástica laboral de acordo com o gênero.

$\mathrm{Na}$ Tabela 3, observa-se que somente as variáveis inatividade física no lazer e consumo abusivo de álcool apresentaram diferença significativa entre os participantes e não participantes da GL. Em ambos os casos, a maior prevalência foi encontrada entre aqueles que referiram não participar das sessões de GL.

A Tabela 4 apresenta a prevalência de indicadores de saúde e do estilo de vida dos trabalhadores, estratificando segundo a participação ou não nas sessões de GL nos homens.

Constatou-se maior prevalência de consumo insuficiente de frutas e de verduras entre os participantes de GL. Nas variáveis inatividade física no lazer, tabagismo e percepção negativa de estresse, maior prevalência foi observada entre os não participantes de GL.

A Tabela 5 demonstra os indicadores de saúde e do estilo de vida dos trabalhadores segundo a participação ou não nas sessões de GL nas mulheres.

Tabela 3.Indicadores de saúde e do estilo de vida dos servidores segundo a participação

\begin{tabular}{|c|c|c|c|c|c|}
\hline \multirow[t]{2}{*}{ Variável } & \multicolumn{2}{|c|}{ Participa } & \multicolumn{2}{|c|}{ Não Participa } & \multirow[t]{2}{*}{$\mathrm{p}$} \\
\hline & $\mathrm{n}$ & $\%$ & $\mathrm{n}$ & $\%$ & \\
\hline Consumo insuficiente de frutas & 79 & 52,3 & 89 & 48,6 & 0,288 \\
\hline Consumo insuficiente de verduras & 51 & 33,8 & 49 & 26,8 & 0,102 \\
\hline Inatividade física no lazer & 73 & 48,3 & 116 & 63,4 & 0,004 \\
\hline Fumante & 18 & 11,9 & 28 & 15,3 & 0,233 \\
\hline Consumo abusivo de álcool & 26 & 17,2 & 47 & 25,8 & 0,039 \\
\hline Percepção negativa saúde & 31 & 20,7 & 33 & 18,1 & 0,328 \\
\hline Percepção negativa de estresse & 26 & 17,3 & 41 & 22,5 & 0,150 \\
\hline Percepção negativa do sono & 26 & 17,3 & 41 & 22,5 & 0,150 \\
\hline Insatisfeitos com colegas de trabalho & 3 & 2,0 & 11 & 6,0 & 0,058 \\
\hline
\end{tabular}

Tabela 4.Indicadores de saúde e do estilo de vida dos homens.

\begin{tabular}{|c|c|c|c|c|c|}
\hline \multirow[t]{2}{*}{ Variável } & \multicolumn{2}{|c|}{ Participa } & \multicolumn{2}{|c|}{ Não Participa } & \multirow[t]{2}{*}{$\mathrm{p}$} \\
\hline & $\mathrm{n}$ & $\%$ & $\mathrm{n}$ & $\%$ & \\
\hline Consumo insuficiente de frutas & 44 & 72,1 & 51 & 52,6 & 0,011 \\
\hline Consumo insuficiente de verduras & 30 & 49,2 & 29 & 29,9 & 0,012 \\
\hline Inatividade física no lazer & 26 & 42,6 & 63 & 64,9 & 0,005 \\
\hline Fumante & 3 & 4,9 & 17 & 17,5 & 0,016 \\
\hline Consumo abusivo de álcool & 18 & 29,5 & 34 & 35,4 & 0,278 \\
\hline Percepção negativa de saúde & 10 & 16,7 & 16 & 16,7 & 0,583 \\
\hline Percepção negativa de estresse & 4 & 6,7 & 17 & 17,7 & 0,039 \\
\hline Insatisfeitos com colegas de trabalho & 1 & 1,7 & 3 & 3,1 & 0,501 \\
\hline
\end{tabular}

Tabela 5. Indicadores de saúde e do estilo de vida dos mulheres.

\begin{tabular}{|c|c|c|c|c|c|}
\hline \multirow[t]{2}{*}{ Variável } & \multicolumn{2}{|c|}{ Participa } & \multicolumn{2}{|c|}{ Não Participa } & \multirow[t]{2}{*}{$\mathrm{p}$} \\
\hline & $\mathrm{n}$ & $\%$ & $\mathrm{n}$ & $\%$ & \\
\hline Consumo insuficiente de frutas & 35 & 38,9 & 38 & 44,2 & 0,288 \\
\hline Consumo insuficiente de verduras & 21 & 23,3 & 20 & 23,3 & 0,566 \\
\hline Inatividade física no lazer & 47 & 52,8 & 53 & 61,6 & 0,134 \\
\hline Fumante & 15 & 16,7 & 11 & 12,8 & 0,305 \\
\hline Consumo abusivo de álcool & 8 & 8,9 & 13 & 15,1 & 0,149 \\
\hline Percepção negativa de saúde & 21 & 23,3 & 17 & 19,8 & 0,348 \\
\hline Percepção negativa de estresse & 22 & 24,4 & 24 & 27,9 & 0,363 \\
\hline Insatisfeitos com colegas de trabalho & 2 & 2,2 & 8 & 9,3 & 0,043 \\
\hline
\end{tabular}


Entre as mulheres, apenas na variável insatisfação com os colegas de trabalho foi a que mostrou diferença significativa, sendo melhor a percepção entre as participantes de GL.

\section{DISCUSSÃO}

Estudos enfocando comportamentos relacionados à saúde ganharam importância quando constatou-se que parte importante das mortes prematuras e das morbidades, estão associadas ao modo como as pessoas vivem ${ }^{13,21}$. Desde então, estudos sobre a prevalência de comportamentos relacionados à saúde em diversos contextos passaram a ser elaborados. A realização destes justifica-se pelo fornecimento de elementos para a efetivação de estratégias que auxiliem as pessoas a adotarem comportamentos considerados mais positivos do ponto de vista da saúde.

Quando comparadas as prevalências dos indicadores de homens e mulheres, constata-se que os homens participam menos da GL, tem consumo insuficiente de frutas e verduras superior às mulheres, bem como o consumo abusivo de bebidas alcoólicas. No entanto, as mulheres apresentaram maior percepção negativa de estresse. Essas diferenças comportamentais entre os gêneros já foram observadas em estudos anteriores ${ }^{14-18}$. Em função destas diferenças comportamentais entre os gêneros, tanto no âmbito regional ${ }^{15,16,18}$ quanto no âmbito nacional, ${ }^{14,17}$ o governo federal criou uma política nacional de atenção primária ao homem, com o objetivo de melhorar o autocuidado e conscientizar esta população do direito à saúde pública, inclusive, tentando aumentar a adesão dos homens às diferentes estratégias de prevenção de doença ${ }^{23}$.

Quando comparada a prevalência dos indicadores segundo participação na GL, verificou-se diferença significativa nas variáveis: Inatividade física no lazer e no consumo abusivo de álcool. Estratificando por gênero, poucas diferenças foram encontradas na prevalência dos indicadores entre mulheres participantes e não participantes da GL.

Por outro lado, entre os homens, importantes diferenças foram evidenciadas. $O$ consumo insuficiente de frutas e verduras foram mais prevalentes entre os trabalhadores participantes da GL. Essa constatação reforça a ideia de que a GL precisa se preocupar, também, com outros comportamentos relacionados à saúde (e não apenas com a atividade física). Segundo a Organização Mundial da Saúde (OMS), o consumo de frutas e verduras é um importante fator preventivo de doenças crônicas não transmissíveis (DCNT) ${ }^{14,17,22,24}$.
A prevalência de inatividade física no lazer foi maior entre os homens que não participam da GL. Dado o corte transversal do estudo, não se pode inferir que a GL é a responsável pela menor prevalência de inatividade física no lazer entre os seus participantes. É possível que haja maior aderência à GL entre aqueles sujeitos ativos fisicamente no tempo livre.

A percepção negativa de estresse foi mais de duas vezes superior entre os trabalhadores não participantes da GL. A principal queixa dos trabalhadores que não participam da GL é não poder parar as tarefas para participar da GL (dados coletados, mas não apresentados no presente estudo). Essa pausa no trabalho, juntamente com a atividade física, pode ser importante para um melhor controle do estresse por parte dos trabalhadores. Entretanto, assim como no caso da inatividade física no lazer, não se pode inferir que de fato seja a GL a responsável pela melhor percepção de estresse, uma vez que é bastante plausível que aqueles com melhor percepção de estresse tenham maior disposição para participarem das sessões.

Os homens participantes da GL também apresentaram menor prevalência de consumo abusivo de bebidas alcoólicas. Apesar de o consumo por si só não ser necessariamente negativo para a saúde, sabe-se que o consumo abusivo deste produto tende a ser prejudicial, podendo causar dependência e gerar problemas familiares, sociais ${ }^{15}$.

Alguns dados aqui encontrados são complexos e necessitariam de um maior suporte da literatura. Entretanto, não foram encontrados estudos que verificassem os comportamentos relacionados à saúde entre participantes e não participantes de GL em outros contextos. Em março de 2010, foi efetuada uma busca no Scielo, utilizando a expressão "ginástica laboral" e nenhum artigo foi encontrado. Essa busca evidencia que existe pouca publicação nas revistas nacionais sobre esse tema, apesar de ser um tema bastante discutido nos cursos da Educação Física e Fisioterapia. Desse modo, pode-se considerar que este estudo apresenta uma contribuição original para a discussão da GL enquanto estratégia de saúde do trabalhador.

A presente pesquisa pode ser utilizada pelos gestores do programa de GL da universidade investigada para adicionar novas intervenções, como palestras educativas, panfletos informativos e até usar o espaço da GL para conversar com os trabalhadores a respeito desses dados. Um estudo longitudinal poderia indicar a efetividade do programa de GL em diferentes desfechos de saúde e, também, poderia 
analisar a influência da GL nos comportamentos relacionados à saúde em longo prazo.

\section{CONCLUSÃO}

Dos comportamentos relacionados à saúde investigados, observou-se que os participantes de GL apresentaram menor prevalência de inatividade física no lazer e consumo abusivo de álcool do que seus pares não participantes.

Quando à análise, foi realizada estratificando-se por gênero, observou-se que as mulheres apresentavam comportamentos relacionados à saúde, semelhantes, independentemente da participação ou não no programa. Já entre os homens, diferenças significativas foram observadas, entre participantes e não participantes da GL, sendo que em dois indicadores (consumo de frutas e verduras) a maior prevalência de consumo inadequado foi entre os não participantes (diferentemente de outros três comportamentos, onde os que participavam da GL tinham melhores indicadores).

A partir dos achados deste estudo, sugere-se a ampliação das estratégias voltadas à saúde do trabalhador no contexto investigado. Parte das recomendações poderia, inclusive, ser adotada pelo programa de GL, ou mesmo poderiam servir de base para a formulação de uma política mais ampliada, que enfoque diversos comportamentos relacionados à saúde, e mesmo em outros determinantes de saúde destes trabalhadores. Entre os possíveis aspectos a serem destacados estão: aumento no nível de conhecimento sobre os benefícios do consumo de frutas e verduras, facilitação no acesso ao consumo destes produtos, conscientização sobre o consumo abusivo de álcool (que nem sempre é interpretado pelos sujeitos como um importante problema de saúde); incentivo à prática de atividade física no tempo livre, fortalecimento das estratégias que auxiliam os trabalhadores a pararem de fumar, entre outras. Para tanto, destaca-se que ações em diferentes frentes, e envolvendo diversos setores, se fazem necessárias. Assim, as estratégias de saúde do trabalhador deveriam ser mais amplas do que somente as sessões de GL, que seriam parte integrante de uma política mais abrangente que buscasse a valorização da saúde dos trabalhadores.

\section{REFERÊNCIAS BIBLIOGRÁFICAS}

1. Alvarez BR. Estilo de vida e hábitos de lazer de trabalhadores, após dois anos de aplicação de um programa de GL e saúde caso - intelbras. [Tese (Doutorado) - Programa de Pós-Graduação em Engenharia de Produção] Florianópolis (SC). Universidade Federal de Santa Catarina; 2002.
2. Martins CO, Duarte MFS. Efeitos da ginástica laboral em trabalhadores da reitoria da UFSC. Rev Bras Ciên Mov 2000;8(4):7-13.

3. Martins CO, Michels G. Saúde X Lucro: Quem ganha com um programa de promoção da saúde do trabalhador? Rev Bras Cineantropom Desempenho Hum 2001;3(1):95-101.

4. Militão AG. A influência da GL para a saúde dos trabalhadores e sua relação com os profissionais que a orientam. [Dissertação (Mestrado) - Programa de Pós-Graduação em Engenharia de Produção] Florianópolis (SC). Universidade Federal de Santa Catarina. Florianópolis; 2001.

5. Martins GC, Barreto SMG. Vivências de ginástica laboral e melhoria da qualidade de vida do trabalhador: resultados apresentados por funcionários adminitrativos do instituto de física da Universidade de São Paulo (Campus São Carlos). Motriz 2007;13(3): 214-24.

6. Carvalho CMC, Moreno CRC. Efeitos de um programa de ginástica laboral na saúde de mineradores. Cad Saúde Coletiva 2007;15(1):117-30.

7. Dishman RK, Dejoy DM, Wilson MG, Vandenberg RJ. Move to improve: A randomized workplace trial to increase physical activity. Am J Prev Med 2009;36(2):133-41.

8. Santos ZMSA, Lima HP. Tecnologia educativa em saúde na prevenção da hipertensão arterial em trabalhadores: análise das mudanças no estilo de vida. Texto Contexto-enferm 2008;17(1):90-7.

9. Charbotel B, Croidieu S, Vohito M. Guerin AC, Renaud L, Jaussaud J. et al. Working conditions in call-centers, the impact on employee health: a transversal study. Part II. Int Arch Occup Environ Health 2009; 82(6):747-56.

10. Dishman RK, Vandenberg RJ, Motl RW, Wilson MG, Dejoy DM. Dose relations between goal setting, theory-based correlates of goal setting and increases in physical activity during a workplace trial. Health Educ Res 2009;1(1):1-12.

11. Lima V. Ginástica Laboral: atividade física no ambiente de trabalho. São Paulo: Phorte, 2003.

12. Nahas MV, Barros MVG, Francallacci VL. O pentáculo do bem-estar: base conceitual para a avaliação do estilo de vida de indivíduos e grupos. Rev Bras Ativ Fís Saúde 2000; 5(2):48-59.

13. Nahas MV. Atividade Física, Saúde e Qualidade de Vida. Londina: Midiograf, 2006.

14. Monteiro CA, Malta DC, Moura EC, Moura L, Morais Neto OL, et al. Vigitel Brasil 2009. Vigilância de fatores de risco e proteção para doenças crônicas por inquérito telefônico. Ministério da Saúde, Brasília; 2009.

15. Leite N, Cieslak F, Osiecki ACV, Bizinelli JA, Timossi 1S, Vilela Junior GB. Estilo de vida e prática de atividade física em colaboradores paranaenses. Rev bras qualidade de vida 2009;1(1):01-14.

16. Barros MVG, Nahas MV. Comportamentos de risco, auto-avaliação do nível de saúde e percepção de estresse entre trabalhadores da indústria. Rev Saúde Pública 2001;35(6): 554-63. 
17. SESI. Estilo de vida e hábitos de lazer dos trabalhadores das indústrias brasileiras: relatório geral. Brasília. 2009.

18. Fonseca SA, Blank VLG, Barros MVG, Nahas, MV. Percepção de saúde e fatores associados em industriários de Santa Catarina, Brasil. Cad Saúde Pública 2008;24(3): 567-76.

19. Winter M. Educação em saúde: Programa de ginástica laboral como atividade educativa. [Dissertação (Mestrado) - Programa de Pós-Graduação em Educação nas Ciências] Ijuí (RS) : Universidade Regional do Noroeste do Estado do Rio Grande do Sul; 2008.

20. Longen WC. Ginástica laboral na prevenção de LER/ DORT:Um estudo reflexivo em uma linha de produção. [Dissertação (Mestrado) - Programa de Pós-Graduação em Engenharia de Produção] Florianópolis (SC) : Universidade Federal de Santa Catarina; 2003.

21. Farinatti PT, Ferreira MS. Saúde, promoção da saúde e Educação Física: conceitos e aplicações. Rio de Janeiro: EDUERJ, 2006.
22. World Health Organization. World health report 2002. Reducing risks, promoting healthy life. Geneva: WHO, 2002.

23. Ministério da Saúde. Política nacional de atenção integral a saúde do homem. Brasília, 2009.

24. World Health Organization. Global Strategy on Diet, Physical Activity and Health. 2010; Disponível em:< http://www.who.int/dietphysicalactivity/ publications/ releases/ pr84/en/ > [2010 march 11].

\section{Endereço para correspondência}

Antonio José Grande

Rua Elias Lopes n46, Vila Gomes.

Sorocaba - SP. Brasil

E-mail: grandeto@gmail.com 